\title{
Changes in operative experience after the introduction of residency: an objective comparison of surgical and endoscopic volumes
}

Kherru Sarah $\underline{\operatorname{Sim}}^{1}$, MBBs, Jin Yao $\underline{T e O}^{2}$, MBBs, FRCSEd, Peng Chung $\underline{\text { Cheow }^{2}}$, MBBS, FAMS

INTRODUCTION The SingHealth Residency General Surgery Programme replaced the basic and advanced specialist training (AST) system in Singapore in 2010. The relative merits of both systems continue to be debated, especially the operational readiness of graduating residents. We set out to compare the operative and endoscopic volumes of graduates from the AST system with those from the first graduating batch of the residency programme during their registrar (or equivalent) years, as a reflection of procedural experience gained during traineeship.

METHODS Operative and endoscopic records of four graduating residents and seven AST system trainees were extracted from the cluster-wide Operating Theatre Management system and compared. Surgeries were analysed as registrar-level operations and their corresponding subspecialties.

RESULTS Registrars and senior residents performed a mean of 1,182 and 533 general surgical operations, respectively. Median percentage loss in operative volume was 50.6\% (range 9.6\%-75.5\%). The mean number of total gastroscopies and colonoscopies performed by registrars (total gastroscopy, $n=819$; total colonoscopy, $n=743$ ) and senior residents (total gastroscopy, $n=376$; total colonoscopy, $n=412$ ) indicated a mean loss of $54.1 \%$ and $44.6 \%$, respectively, in gastroscopic and colonoscopic experience.

CONCLUSION The residency programme aims to provide robust and complete surgical training. The operational readiness of its graduates is often scrutinised against that from the old system. Although a significant difference in surgical and endoscopic volumes was observed between the two trainee groups, this is only one marker of surgical experience and technical competence.

Keywords: general surgery, residency and internship, Singapore

\section{INTRODUCTION}

The general surgery training programme in Singapore was revamped in 2010 with the introduction of a residency programme, the SingHealth Residency General Surgery Programme, which emulates the North American model for specialist training. Many differences exist between the old Basic Specialist Training/Advanced Specialist Training (BST/AST) system and the new residency system, and the relative merits of both continue to be a matter of debate.

One of the main differences between the two systems is the entry into and length of training at the registrar or junior specialist level. In the traditional system, a trainee was required to apply for AST and be granted progression into it only on acceptance. The trainee would then undergo four years of AST as a registrar, consisting of six-monthly rotations in various subspecialties of general surgery. Under the residency system, in contrast, progression into senior residency - the registrar equivalent - is guaranteed and not subject to an application process. The senior resident would then undergo two additional years of training with subspecialty rotations of not more than four months each (as per the stipulation of the Accreditation Council for Graduate Medical Education-International [ACGME-I]). ${ }^{(1)}$ Despite their condensed training of half of the original AST duration, senior residents are expected to function at the same level as their AST counterparts and take on equal responsibilities. They continue to be employed as associate consultants upon completion of the senior residency and exit examinations.

Further exacerbating this truncation in training is the implementation of ACGME-I duty hour requirements. Teo et al analysed local data and described a projected $12 \%-22 \%$ loss of operative time annually for a registrar adhering to ACGME-I duty hour requirements. ${ }^{(2)}$

As with any other profession that calls for technical competence, having adequate time and experience are essential not just for learning but also honing the skills necessary for ensuring good outcomes. It is no surprise that concerns have been raised regarding the adequacy of training for our surgeons in the residency programme, given the significant reduction in training time. Hence, with the recent graduation of the first batch of residents in Singapore in 2015, we set out to compare the operative and endoscopic volumes of graduates from the AST system with those from the residency system during their registrar (or equivalent) years, as a reflection of procedural experience and exposure gained during their traineeship.

\section{METHODS}

The operative and endoscopy logs of four residents from the first graduating batch were reviewed for the period between July 2013 and July 2015, which corresponded to their two years as senior

${ }^{1}$ Department of General Surgery, ${ }^{2}$ Department of Hepatopancreatobiliary and Transplant Surgery, Singapore General Hospital, Singapore

Correspondence: Dr Teo Jin Yao, Consultant, Department of Hepatopancreatobiliary and Transplant Surgery, Singapore General Hospital, Outram Road, Singapore 169608. teo.jin.yao@singhealth.com.sg 


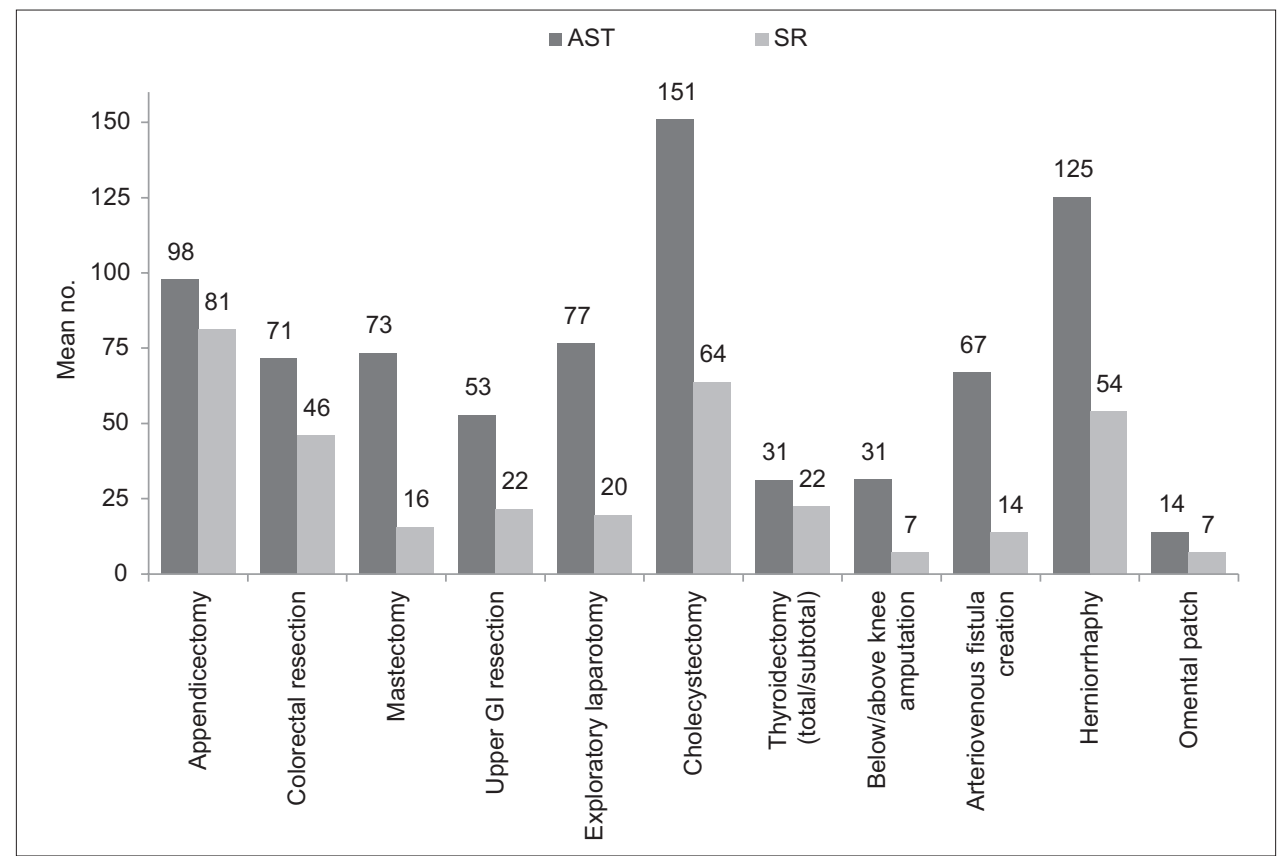

Fig. 1 Chart shows the registrar-level surgeries performed by registrars and senior residents. AST: advanced specialist training; Gl: gastrointestinal; SR: senior resident

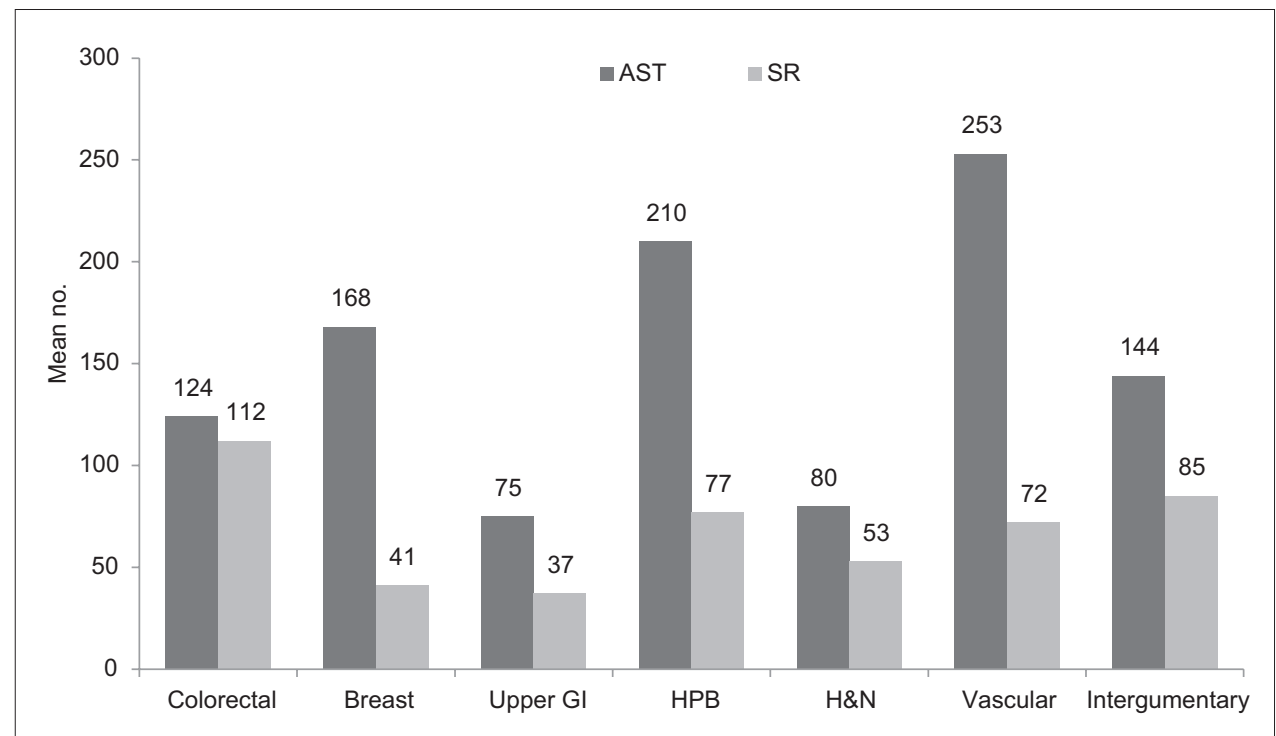

Fig. 2 Chart shows the subspecialty surgeries performed by registrars and senior residents. AST: advanced specialist training; H\&N: head and neck; HPB: hepatopancreatobiliary; GI: gastrointestinal; SR: senior resident

residents. These were compared with the records of seven AST candidates, who commenced AST at the time of inception of residency. Data was extracted from the cluster-wide Operating Theatre Management (OTM) system, thus ensuring that all operations performed in the major operating theatre, ambulatory surgery centre and endoscopy centre were captured.

We examined particular operations that registrars were traditionally expected to be able to carry out independently, namely appendicectomies, hernia repairs and lower limb amputations. The operations were also categorised into their corresponding subspecialties to evaluate total volume as a reflection of the trainee's experience in the various subspecialties. The logs were further examined to record and analyse the number and percentage of operations that were specifically performed as first surgeon.
The endoscopy volumes were also analysed in both groups and further categorised into diagnostic and therapeutic endoscopies. No statistical analysis was performed, as due to the inherent difference in training time, any difference seen in the results would not be due to chance.

\section{RESULTS}

Registrars from the AST system performed a mean number of 1,182 general surgical operations as compared to 533 by senior residents. Fig. 1 shows a breakdown of the mean number of registrarlevel operations in which AST registrars and senior residents participated. Fig. 2 displays the mean number of these operations according to the various subspecialties in general surgery. The discrepancies in operative volume between the two groups were 


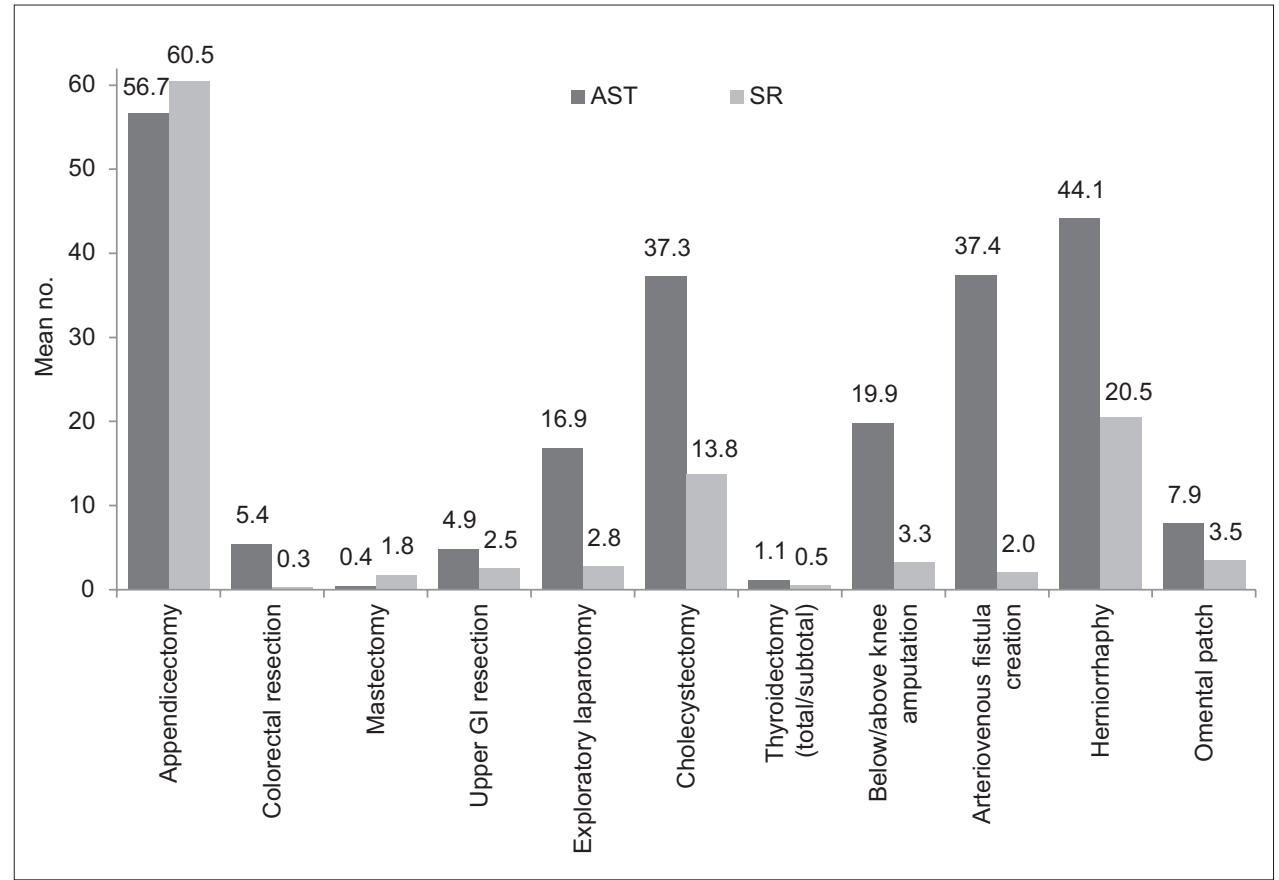

Fig. 3 Chart shows the registrar-level surgeries performed by registrars and senior residents as first surgeon. AST: advanced specialist training; GI: gastrointestinal; SR: senior resident

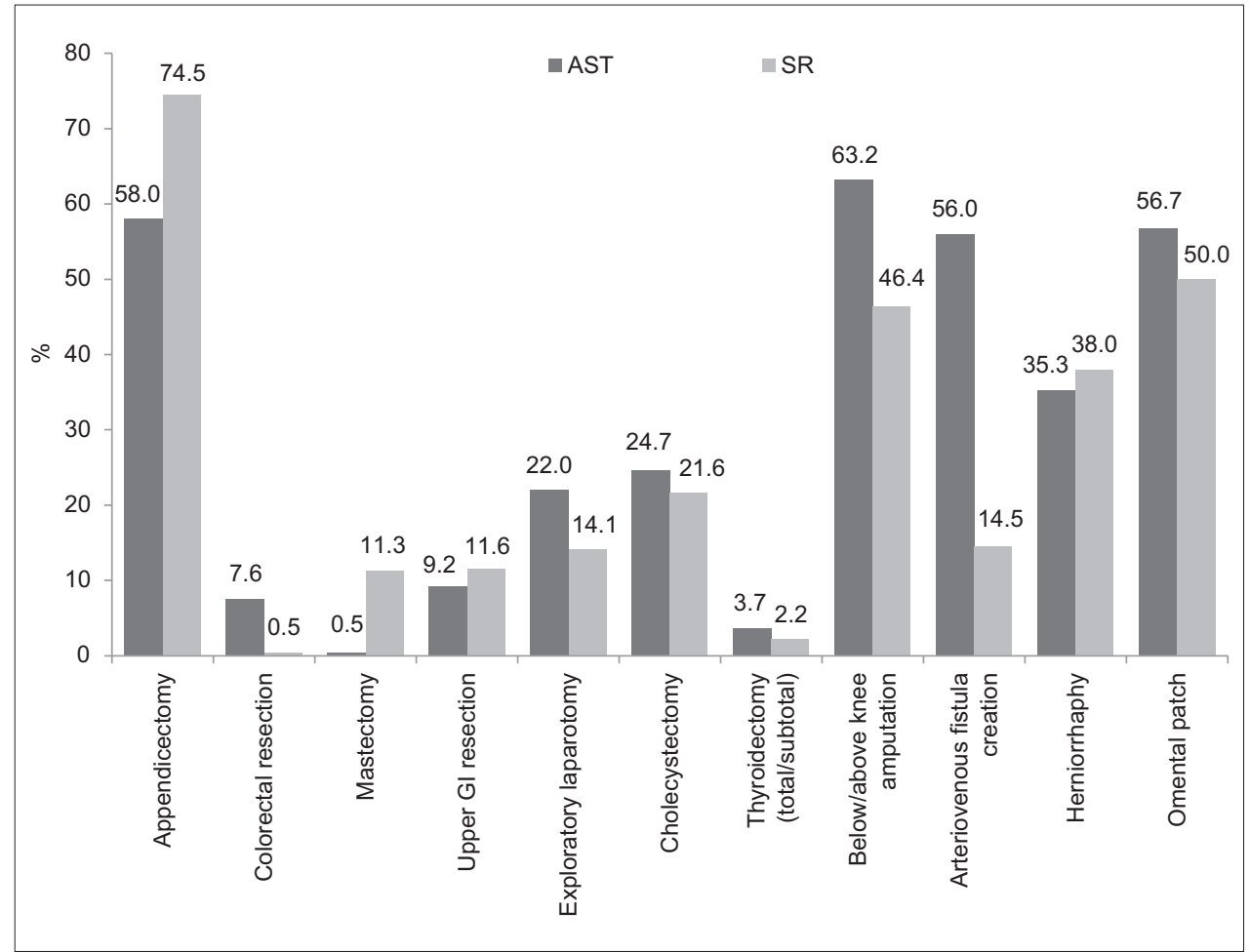

Fig. 4 Chart shows the proportions of operations performed by registrars and senior residents as first surgeon. AST: advanced specialist training; Gl: gastrointestinal; SR: senior resident

generally consistent regardless of whether registrar-level skills or subspecialty experience were being tested. The median percentage loss in operative volume was $50.6 \%$ (range $9.6 \%-75.5 \%$ ).

Fig. 3 shows a comparison of the mean number of registrarlevel operations performed by registrars and senior residents as first surgeon, according to OTM records, between the two groups. The differences in operative volume as first surgeon appeared to be even starker than those in total operative participation, with the senior residents performing less than half of the first-surgeon operations when compared to AST registrars for most registrarlevel operations. With the exception of appendicectomies and mastectomies, the median percentage loss in first-surgeon operative volume was $63.0 \%$ (range $49.0 \%-94.7 \%$ ).

Operations performed by registrars and senior residents as first surgeon, as a percentage of their total operative participation, are shown in Fig. 4. We found that of the total number of operations 


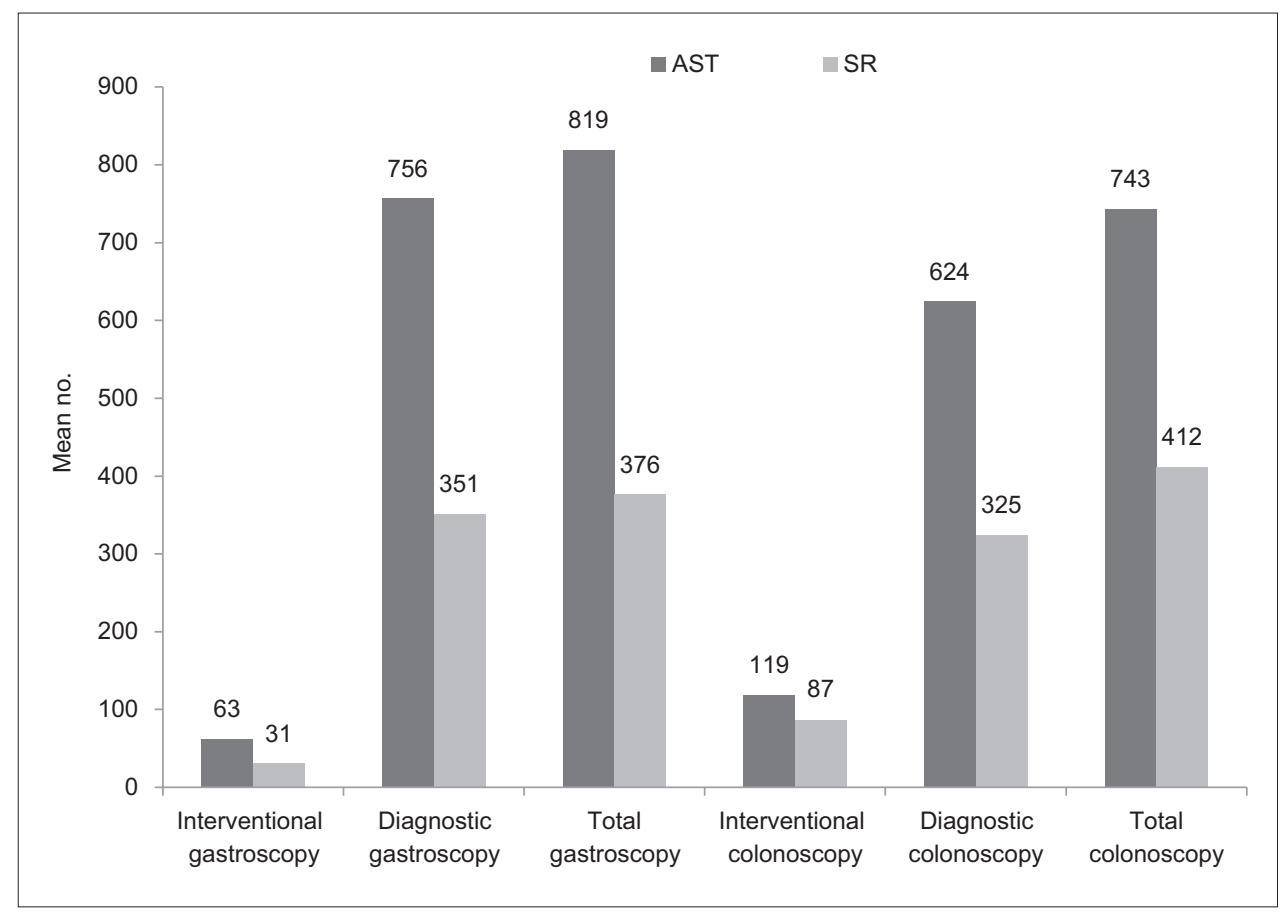

Fig. 5 Chart shows the endoscopies performed by registrars and senior residents. AST: advanced specialist training; SR: senior resident

in which a trainee participated, senior residents were first surgeons for a higher proportion of appendicectomies, mastectomies, upper gastrointestinal resections and herniorrhaphies, but a lower proportion of colorectal resections, exploratory laparotomies, cholecystectomies, thyroidectomies (total/subtotal), lower limb amputations, arteriovenous fistula creations and omental patch repairs.

Endoscopy volumes (Fig. 5) revealed consistent differences between the two groups, as the mean number of total gastroscopies and colonoscopies performed by the registrars (total gastroscopy, $\mathrm{n}=819$; total colonoscopy, $\mathrm{n}=743$ ) and senior residents (total gastroscopy, $\mathrm{n}=376$; total colonoscopy, $\mathrm{n}=412$ ) indicated a mean loss of $54.1 \%$ and $44.6 \%$, respectively, in gastroscopic and colonoscopic experience.

\section{DISCUSSION}

It is unsurprising that the operative and scope numbers of senior residents over two years would be less than those of AST registrars over four years. Initial proponents of the residency system believed that more structured, dedicated and supervised training would make up for the reduced number of years, so that the end result in terms of competence would be similar for both programmes. For example, under the BST system, a trainee did postings for a minimum of 24 months (with almost all trainees accumulating at least three years' worth of postings in practice), many of which may not be relevant, while junior residents undergo 36 months of dedicated, structured postings. It is also argued that junior residents may start attending cases earlier, under closer supervision, so that senior residents may possibly start off 'better' than registrars. Additionally, the residency curriculum incorporates greater use of simulation training. During the senior resident years, because residents and the faculty know that they have only two years to train, residents may be allowed to do more during surgery and hence, may benefit more from each patient attended.

However, given the significant cuts in training time between the traditional system and the residency training programme, one must question the adequacy of the latter in equipping trainees with sufficient experience. In this study, we examined the effects of the shortened training time on surgical volume among the very first batch of graduating residents. Our results, showing up to a $75.5 \%$ loss in surgical volume, raise concerns regarding the preparedness of trainees graduating from the residency programme. A recent study analysing surgical volumes in orthopaedic residents found significant variability among them, with residents in the 10th percentile logging significantly fewer procedures than those in the 90th percentile. ${ }^{(3)}$ This suggests that the deficit in operative training volume may be even more worrying among graduates of senior residency, as those in the lower percentiles would have even less operative experience than their peers in the higher percentiles at the end of their training.

As registrars progress in their training, they may take a lead role in a greater proportion of surgeries or even perform the bulk of the operation independently. Certainly, operative independence is an essential skill when on call and operating on emergency patients. A 50\% reduction in training duration might result in a disproportionate loss of surgical independence. One encouraging finding was that, compared to AST registrars, senior residents performed a higher proportion of total operations as first surgeon in certain operations that are commonly performed emergently overnight (e.g. appendicectomies and upper gastrointestinal resections), although the mean number of operations performed as first surgeon was still generally higher among AST registrars. However, this trend was not reflected in other common emergency operations, such as exploratory laparotomies and omental patch repairs. It should be emphasised that, as described previously, the 
absolute numbers of surgeries and first-surgeon operations were significantly lower among the senior residents. We recognise that the OTM listing of surgeons, for a variety of reasons, may not accurately reflect the actual role played by the trainee during an operation, but for the purposes of this study, it was assumed that a trainee listed as first surgeon would have taken the lead for the majority of the operation. Hence, although the aforementioned trend was not seen in all registrar-level operations, it may continue to grow in the subsequent batches of senior residents as a way to make up for the losses in total operative volume.

With regard to endoscopic numbers, AST registrars expectedly logged significantly more patient cases than senior residents. However, in terms of objective competence, senior residents still managed to reach quantities sufficient to obtain accreditation in either of the tertiary hospitals under SingHealth - Singapore General Hospital (200 gastroscopes and 100 colonoscopes) and Changi General Hospital (300 gastroscopes and 100 colonoscopes).

A survey distributed to members of the American College of Surgeons revealed that only $53 \%$ of surgeons aged 45 years and above believed that the residency programme was adequate for preparing trainees to function independently after graduation. ${ }^{(4)}$ This is in spite of the fact that the residency programme is more well established in North America. We should therefore be prepared to closely examine the operational readiness of our local graduates.

Notably, it is currently uncertain whether a reduction in operative experience translates to differences in clinical outcomes. Operative volume is only a surrogate marker of the adequacy of one's training, and other factors (e.g. exposure to a breadth of cases, perioperative management, clinical acumen and decision-making) all contribute to clinical outcomes. We acknowledge that this represents the main flaw in our study. However, by relying on data extracted from the cluster-wide OTM system, we have obtained a reliable and robust dataset, free from the reporting and/or recall bias that would be inherent if self-recorded logbooks were used. Therefore, it can be said that this study manages to objectively quantify the difference in procedural volumes logged by registrars and senior residents over the relevant time periods of training.

Indeed, further studies focusing on comparisons of patient outcomes and clinical competence between the two groups are warranted. However, such studies may be difficult, if not nearly impossible, to conduct in an unbiased fashion. Currently, the AST system is still in the process of being phased out; at the same time, the management of a majority of complicated cases is currently being led by more experienced and senior surgeons who were trained and honed in the original system. The true effect of having less experienced trainees is thus difficult to determine. However, for the good of our patients, we should acknowledge these differences in training and experience before their effect becomes clinically apparent. Perhaps minimum numbers could be met in order for trainees to obtain accreditation for registrar-level operations, and a requirement of being certified fit to practise by supervising faculty should be adopted as a way to objectively judge competence, similar to what has been established for endoscopic accreditation.

In conclusion, the residency programme aims to provide robust and structured surgical training. However, in its replacement of an otherwise reliable and stable training programme in Singapore, we are inevitably led to compare the two and how their products - the graduates - may differ. At first glance, the significant differences in operative and endoscopic experience presented in this study raise concerns about the preparedness of the new residency graduates to function as independently as their AST counterparts had in the past. However, we acknowledge that surgical volume alone is not a perfect surrogate for clinical and technical competence. While the implications of a shortened training are not immediately apparent and may be debatable, it would nonetheless be prudent to anticipate such eventualities in order to prepare for the challenges ahead, given the changing landscape of general surgery training in Singapore, so as to ensure the continuity of adequately prepared surgeons for generations to come.

\section{REFERENCES}

1. ACGME International. Advanced Specialty Program Requirements for Graduate Medical Education in General Surgery. Available at: http://www. acgme-i.org/Portals/0/Specialties/GeneralSurgery/GeneralSurgery07012016. pdf?ver=2017-09-18-093848-387. Accessed December 17, 2017.

2. Teo JY, Lee SY, Chua AJ, Wong WK. Will the local ACGME-trained surgeon be adequately prepared? An estimate of the impact of duty hour restrictions on operative experience. Ann Acad Med Singapore 2013; 42:203-6.

3. Gil JA, Daniels AH, Weiss AP. Variability in surgical case volume of orthopaedic surgery residents: 2007 to 2013. J Am Acad Orthop Surg 2016; 24:207-12.

4. Napolitano LM, Savarise M, Paramo JC, et al. Are general surgery residents ready to practice? A survey of the American College of Surgeons Board of Governors and Young Fellows Association. J Am Coll Surg 2014; 218:1063-1072.e31. 